\title{
RCW 86, an extended SNR viewed at high energy with the new Fermi-LAT Pass 8 event reconstruction
}

\author{
Benjamin Condon ${ }^{* \dagger}$, Marianne Lemoine-Goumard \\ Universite Bordeaux 1, CNRS/IN2P3, Centre d'Etudes Nucleaires de Bordeaux Gradignan, \\ 33175 Gradignan, France \\ E-mail: condon@cenbg.in2p3.fr, lemoine@cenbg.in2p3.fr
}

\section{Micaela Caragiulo}

Universita degi Studi di Bari Aldo Moro, INFN, 70121 Bari, Italia

E-mail: micaela.caragiulo@ba.infn.it

\section{John W. Hewitt}

NASA Goddard Space Flight Center, Greenbelt, MD 20771, USA

E-mail: john.w.hewitt@nasa.gov

\begin{abstract}
Supernovae Remnants (SNRs) are thought to be the primary source of galactic cosmic rays observed on Earth. Detected in radio, infrared, X-rays and at high $(\mathrm{GeV})$ and very high energy $(\mathrm{TeV})$ gamma rays, RCW 86 is a good candidate for efficient particle acceleration and might be the remnant of the historical supernova SN 185. Using more than 6 years of data acquired by the Fermi Large Area Telescope with the new Pass 8 event reconstruction, RCW 86 is now detected as a significant extended source at $\mathrm{GeV}$ energies, with a radius of $0.37^{\circ}$. The results of our deep morphological and spectral analysis provide new constraints on the origin of the gamma-ray emission and on key parameters such as the asymmetry of the morphology, the density of the surrounding medium and the total energy in accelerated particles. These new constraints will be presented and discussed in the light of existing estimates.
\end{abstract}

The 34th International Cosmic Ray Conference,

30 July- 6 August, 2015

The Hague, The Netherlands

\footnotetext{
*Speaker.

${ }^{\dagger}$ Fellowship from the Centre National d'Etudes Spatiales (CNES)
} 